\title{
An Optimization Synchronization Algorithm for TDDM Signal
}

\author{
Fang Liu and Yongxin Feng \\ School of Information Science and Engineering, Shenyang Ligong University, Shenyang 110159, China \\ Correspondence should be addressed to Yongxin Feng; onceowned_1019@163.com
}

Received 31 May 2016; Accepted 5 September 2016

Academic Editor: Jit S. Mandeep

Copyright (C) 2016 F. Liu and Y. Feng. This is an open access article distributed under the Creative Commons Attribution License, which permits unrestricted use, distribution, and reproduction in any medium, provided the original work is properly cited.

\begin{abstract}
The time division data modulation (TDDM) mechanism is recommended to improve the communications quality and enhance the antijamming capability of the spread spectrum communication system, which will be used in the next generation global navigation satellite (GNSS) systems. According to the principle and the characteristics of TDDM signal, an optimization synchronization algorithm is proposed. In the new algorithm, the synchronization accuracy and environmental adaptability have been improved with the special local sequence structure, the multicorrelation processing, and the proportion threshold mechanism. Thus, the inversion estimation formula was established. The simulation results demonstrate that the new algorithm can eliminate the illegibility threat in the synchronization process and can adapt to a lower SNR. In addition, this algorithm is better than the traditional algorithms in terms of synchronization accuracy and adaptability.
\end{abstract}

\section{Introduction}

To improve the communications quality and the antijamming capability of the spread spectrum communication system, the time division data modulation (TDDM) [1] mechanism is recommended based on the traditional modulation methods. The new mechanism will be used in the next generation global navigation satellite systems [2, 3], for example, the GPS-III $[4,5]$ system. The difference of the TDDM and the traditional modulation mechanism is that nondata element is permitted in the new signal, and the spread spectrum processing depends on the pseudonoise (PN) code odd or even scheduling. The typical TDDM principle is that the even sequences sign is the same as the PN code sign, whereas the odd sequence sign is uncertain. The uncertain factor will cause some illegibility problems in the signal synchronization processing, such as inconspicuous correlation peak and higher data inverse error.

If the traditional algorithms are applied in TDDM signal synchronization, the correlation peak will decrease to half of the autocorrelation function when the data inversion position is in the middle of the received signal, and the correlation result is the same as the cross-correlation function when the data sign is successive negative. Thus, the approximate noncorrelation characteristic appears in this TDDM signal receiving. Currently, several PN code receiving $[6,7]$ and detection [8] technologies have been proposed, which can provide some referenced methods for TDDM signals synchronization. And the synchronization circuit system [9] based on field programmable gate array has become one of the development directions for GPS and even for a GNSS embedded real-time software receiver. Furthermore, large numbers of synchronization algorithms are proposed to improve the navigation signals receiving capability. For example, in [10], an efficient differential coherent accumulation algorithm for weak GPS signal bit synchronization was presented to reduce the computational load by approximately sixfold. In [11], the authors provided a detailed analysis of the significance of the cell-correlation phenomenon in MF correlates for the two widely used signal families in GNSS, namely, BPSK and BOC; next, the theoretical analysis was validated by Monte Carlo simulations. In [12], the code acquisition architecture for GNSS was presented to reduce the buffer resource required without reducing the numbers of code, frequency, and satellite bins. In [13], the authors proposed to reconstruct the correlation results of DBZP to improve the detection performance of the previously published double-block zero-padding (DBZP) method for weak GNSS long PN code signals. 
In summary, from the perspective of algorithm generality, the TDDM signal synchronization algorithm is usually divided into two categories, namely, the filled zero (FZ) algorithm [14] and the positive-negative (PN) algorithm [15]. The principle of the FZ algorithm is that the odd bits of the local code are replaced by zero, and then the correlation arithmetic is executed by using the preprocessed receiving signal and the local signal. This algorithm reduces the uncertainty factor and enhances the estimation inversion precision by sacrificing the correlation peak in the synchronization processing. The principle of the $\mathrm{PN}$ algorithm is that the local code is processed by the positive and negative TDDM mechanism, and next, the correlation arithmetic is executed by using the preprocessed receiving signal and the positive local sequence. This algorithm imports the inversion judgment mechanism and combines the correlation peaks of the two channels to estimate the inversion position. The FZ algorithm and $\mathrm{PN}$ algorithm can be applied to receive TDDM signal and to estimate the inversion position, but the estimation precision of these algorithms is expected to improve in a complicated environment.

\section{The Optimization Synchronization Algorithm with Illegibility Elimination}

To describe the new algorithm, the digital intermediate frequency received signal $S^{\prime}(n)$ is modeled after frequency downconversion, ADC, and band-pass filtering. The processed signal is expressed as

$$
S^{\prime}(n)=D(n) C_{T}(n) \sin \left(\omega n+\omega_{0} n\right)+N_{0}(n),
$$

where $D(n)$ is the binary data, $C_{T}(n)$ is the TD codes, $\omega$ is the intermediate frequency due to the downconversion, $\omega_{0}$ is the Doppler frequency, and $N_{0}(n)$ is the band-limited mixed noise signals. $L$ is the number of the received signal.

To eliminate the data inversion illegibility threat, we structure the special local sequences. The local sequence is produced using the same principle with the PN code of the received signal, and it is denoted as $C_{i}(n)$. The phase of the local code is expressed as variable $i$, for which the initialization value is set as $p$. Furthermore, the filling zero sequence is produced and expressed as $C_{0 i}(n)$, in which the odd number sequence is zero and the even number sequence is the same as $C_{i}(n)$; these processes are expressed as

$$
\begin{aligned}
C_{0 i}(n) & =\left\{a_{i}, a_{1+i}, a_{2+i}, \ldots, a_{L+i-1}\right\} \\
C_{i}(n) & =\left\{b_{i}, b_{1+i}, b_{2+i}, \ldots, b_{L+i-1}\right\}
\end{aligned}
$$

with

$$
a_{n}= \begin{cases}0, & n=\text { odd number } \\ b_{n}, & n=\text { even number. }\end{cases}
$$

The preprocessing received signal is executed by the in-phase channel and quadrature channel frequency compensation arithmetic to overcome the influence of the uncertain frequency. The processing is expressed as

$$
\begin{aligned}
S_{I}^{\prime}(n) & =S^{\prime}(n) \sin (\omega n) \\
= & {\left[D(n) C_{T}(n) \sin (\omega n+\Delta n)+N_{0}(n)\right] \sin (\omega n) } \\
= & \frac{1}{2} D(n) C_{T}(n) \cos (\Delta n) \\
& -\frac{1}{2} D(n) C_{T}(n) \cos (2 \omega n+\Delta n) \\
& +N_{0}(n) \sin (\omega n), \\
S_{Q}^{\prime}(n)= & S^{\prime}(n) \cos (\omega n) \\
= & {\left[D(n) C_{T}(n) \sin (\omega n+\Delta n)+N_{0}(n)\right] \cos (\omega n) } \\
= & \frac{1}{2} D(n) C_{T}(n) \sin (2 \omega n+\Delta n) \\
& +\frac{1}{2} D(n) C_{T}(n) \sin (\Delta n)+N_{0}(n) \cos (\omega n) .
\end{aligned}
$$

The two-channel compensated signals are processed using low-pass filtering, which are given by

$$
\begin{aligned}
S_{I}(n) & =\frac{1}{2} D(n) C_{T}(n) \cos (\Delta n), \\
S_{\mathrm{Q}}(n) & =\frac{1}{2} D(n) C_{T}(n) \sin (\Delta n) .
\end{aligned}
$$

The local code phase $i$ is moved from $p$ to $p+L-1$, and next, the filling zero sequence $C_{0 i}(n)$ is, respectively, multiplied by the received signal $S_{I}(n)$ and $S_{Q}(n)$. These quantities are given by

$$
\begin{aligned}
X_{I 0}(n) & =S_{I}(n) \cdot C_{0 i}(n) \\
& =\frac{1}{2} D(n) C_{T}(n) C_{0 i}(n) \cos (\Delta n), \\
X_{\mathrm{Q} 0}(n) & =S_{\mathrm{Q}}(n) \cdot C_{0 i}(n) \\
& =\frac{1}{2} D(n) C_{T}(n) C_{0 i}(n) \sin (\Delta n) .
\end{aligned}
$$

Combining the product results of the two channels, the square sum is calculated to inhibit the Doppler influence and then to achieve the correlation purpose. The processing is expressed as

$$
\begin{aligned}
X_{i} & =\sum\left(X_{I 0}(n)\right)^{2}+\left(X_{\mathrm{Q} 0}(n)\right)^{2} \\
& =\sum\left[\left(\frac{1}{2} D(n) C_{T}(n) C_{0 i}(n) \cos (\Delta n)\right)^{2}\right. \\
& \left.+\left(\frac{1}{2} D(n) C_{T}(n) C_{0 i}(n) \sin (\Delta n)\right)^{2}\right]
\end{aligned}
$$




$$
\begin{aligned}
& =\sum\left[\frac{1}{4}\left(D(n) C_{T}(n) C_{0 i}(n)\right)^{2}\right. \\
& \left.\cdot\left(\cos (\Delta n)^{2}+\sin (\Delta n)^{2}\right)\right] \\
& =\sum\left[\frac{1}{4}\left(D(n) C_{T}(n) C_{0 i}(n)\right)^{2}\right] .
\end{aligned}
$$

From (7), we can see that the influences of Doppler and wave have been restrained. Furthermore, the correlation results $X_{i}$ are obtained using the change of variable $i$, so the correlation sequence is expressed as

$$
X(n)=\left\{X_{p}, X_{p+1}, X_{p+2}, \ldots, X_{p+L-1}\right\} .
$$

Although the existing algorithms can automatically adjust the threshold, they cannot improve the correlation peak values. Thus, we structure the proportion peak to improve the new algorithm adaptability. We define the proportion peak $V$ as

$$
V=\frac{\max [X(n)]}{\sum(X(n))} .
$$

Thus, the peak judgment operation is performed after the proportion peak processing. If the proportion peak cannot exceed the threshold $G$, then reacquisition will be operated after the local PN codes and adjusted Doppler shift, or else the value $i$ of $X_{i}$ is calculated using the proportion peak $V$. Let the calculated $i$ be $P_{M}$; namely, $i=P_{M}$. Thus, the calculated $i$ is imported to formula (2), and the results are expressed as

$$
\begin{aligned}
C_{0 P_{M}}(n) & =\left\{a_{P_{M}}, a_{1+P_{M}}, a_{2+P_{M}}, \ldots, a_{L+P_{M}-1}\right\}, \\
C_{P_{M}}(n) & =\left\{b_{P_{M}}, b_{1+P_{M}}, b_{2+P_{M}}, \ldots, b_{L+P_{M}-1}\right\} .
\end{aligned}
$$

The received signal $S_{I}(n)$ is multiplied by the new local signals $C_{0 P_{M}}(n)$ and $C_{P_{M}}(n)$, whose results are given by (11) and (12). Thus, the accumulative totals are given by (13) and (14):

$$
\begin{aligned}
X_{I 0}^{\prime}(n) & =S_{I}(n) \cdot C_{0 P_{M}}(n) \\
& =\frac{1}{2} D(n) C_{T}(n) C_{0 P_{M}}(n) \cos (\Delta n), \\
X_{I Z}^{\prime}(n) & =S_{I}(n) \cdot C_{P_{M}}(n) \\
& =\frac{1}{2} D(n) C_{T}(n) C_{P_{M}}(n) \cos (\Delta n), \\
R_{I 0} & =\sum\left(X_{I 0}^{\prime}(n)\right) \\
& =\frac{1}{2} \sum\left(D(n) C_{T}(n) C_{0 P_{M}}(n) \cos (\Delta n)\right), \\
R_{I Z} & =\sum\left(X_{I Z}^{\prime}(n)\right) \\
& =\frac{1}{2} \sum\left(D(n) C_{T}(n) C_{P_{M}}(n) \cos (\Delta n)\right) .
\end{aligned}
$$

In this condition, let $A$ be the correlation result of the even number sequence when the data sign is negative, and let $\alpha$ be the influence coefficient, which is given by (15). At the same time, let $B$ be the correlation result of the even number sequence when the data sign is positive, and let $\beta$ be the influence coefficient, which is given by (16):

$$
\begin{aligned}
& A=\frac{1}{2 \beta} \sum\left(D(n) C_{T}(n) C_{0 P_{M}}(n) \cos (\Delta n)\right), \\
& \text { when the data sign is negative, } \\
& B=\frac{1}{2 \alpha} \sum\left(D(n) C_{T}(n) C_{0 P_{M}}(n) \cos (\Delta n)\right), \\
& \text { when the data sign is positive. }
\end{aligned}
$$

In this paper, the theoretical correlation result between the local sequence and the received signal is defined as

$$
R_{I 0}^{\prime}=\sum\left(D(n) C_{T}(n) C_{0 P_{M}}(n)\right) .
$$

In mixed noise condition, the relationship between the correlation result and $A, B, \alpha$, and $\beta$ can been given by

$$
\begin{aligned}
& \alpha \cdot B+\beta \cdot A=R_{I 0}, \\
& \alpha \cdot B-\beta \cdot A=R_{I Z}-R_{I 0} .
\end{aligned}
$$

In view of these characteristics and combining (18) and (19), we can calculate the sequence number of the same sign as

$$
B=\frac{R_{I Z}}{2 \alpha}
$$

Furthermore, the relationship between the real correlation and the theoretical correlation result is given by (21), and then the influence coefficient $\alpha$ is calculated and given by (22):

$$
\begin{gathered}
R_{I 0}=\alpha \cdot R_{I 0}^{\prime}, \\
\alpha=\frac{R_{I 0}}{R_{I 0}^{\prime}} .
\end{gathered}
$$

We can rectify formula (20) as (23), and then we obtain the formula (24), according to the relationship of the theoretical correlation result and the processing number. Thus, formula (23) is renewed as (25):

$$
\begin{aligned}
B & =\frac{R_{I Z}}{2} \cdot \frac{R_{I 0}^{\prime}}{R_{I 0}}=\frac{R_{I Z} \cdot R_{I 0}^{\prime}}{2 R_{I 0}}, \\
R_{I 0}^{\prime} & =\frac{L}{2}, \\
B & =\frac{R_{I Z} \cdot L}{4 R_{I 0}} .
\end{aligned}
$$

In view of the same relation of the odd number and the even number, the sum of the positive sign and the negative sign is equal to the processing number $L$. Finally, the inversion estimation formula is established to calculate 


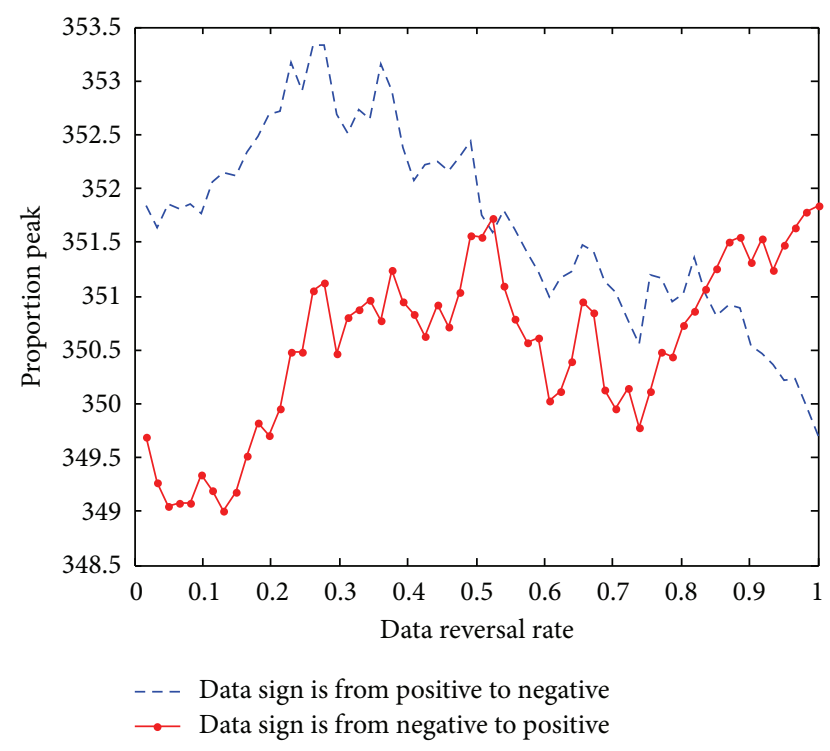

FIGURE 1: The proportion peak change with changing data reversal rate.

the reversal position combining the above formulas, and the estimation reversal position is expressed as

$$
\rho=\frac{L-2 B}{L}=1-\frac{R_{I Z} \cdot L}{2 R_{I 0} \cdot L}=1-\frac{R_{I Z}}{2 R_{I 0}} .
$$

\section{Simulation and Analysis}

3.1. The Capability Analysis. To furnish the judgment criterion for the synchronization algorithms, the thresholds $G$ for the new algorithm and the existing algorithms are ascertained. These algorithms are analyzed using the following parameters: $5 \mathrm{MHz}$ PN code frequency, $10 \mathrm{MHz}$ intermediate wave frequency, $40 \mathrm{MHz}$ sampling frequency, and $50 \mathrm{bps}$ data rate. In this simulation condition, three algorithms' thresholds are as high as 10 .

The ratio of the actual inversion position to the total length is defined as the data reversal rate. And with changing data reversal rate, the synchronization proportion peak change is shown in Figure 1. The results show that the new algorithm proportion peak can exceed 10 and can achieve the threshold required when the data sign is from positive to negative or is from negative to positive. With changing data reversal rate, the changes of the estimated reversal position and the theoretical reversal position are shown in Figure 2, the results indicate that the estimated value is approximately equal to the theoretical value. Furthermore, there may be some error between the estimated inversion position and the actual inversion position, so the estimated reversal error is tested. With changing actual data reversal rate, the estimated reversal error change is shown in Figure 3, in which $x$-axis is the ratio of the actual inversion position to the total length and $y$-axis is the estimated error between the estimated inversion position and the actual inversion position. The results show that the error is highest when the actual data

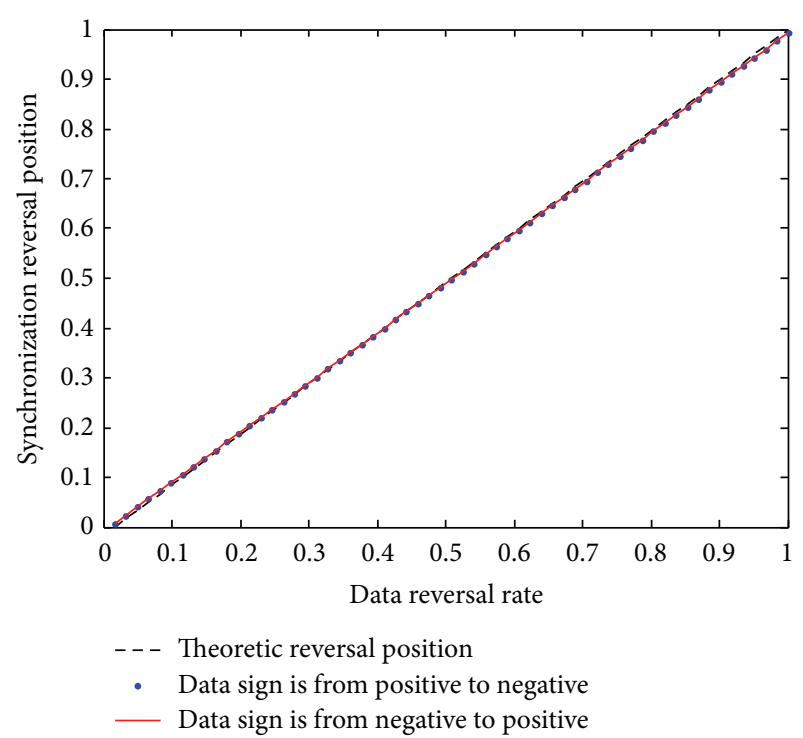

FIGURE 2: The reversal position change with changing data reversal rate.

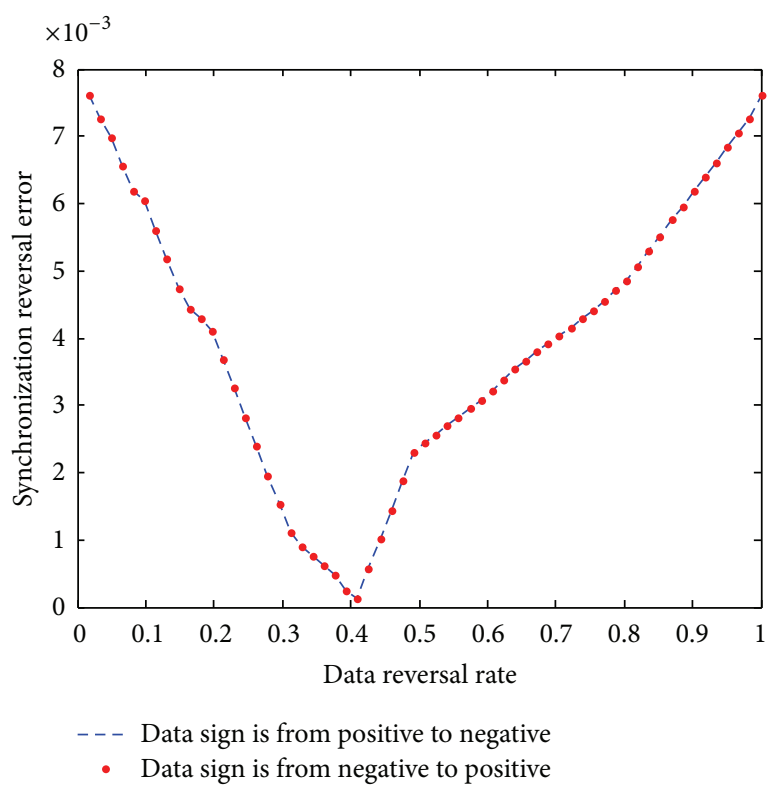

FIgURE 3: The estimated reversal error change with changing data reversal rate.

reversal position is in the middle of the received signal; however, all errors can be less than the $10^{-4}$ level.

In the conditions that the data sign is from positive to negative or is from negative to positive, the relationship of the synchronization proportion peak and SNR is shown in Figure 4, revealing that the proportion peak decreases gradually with decreasing SNR. The proportion peak cannot achieve the threshold required when the SNR is less than $-37 \mathrm{~dB}$; thus, the new algorithm's adaptability to the SNR environment is more than $-37 \mathrm{~dB}$.

Furthermore, when the input parameter of the actual reversal rate is constant, which is 0.32 , the new algorithm 


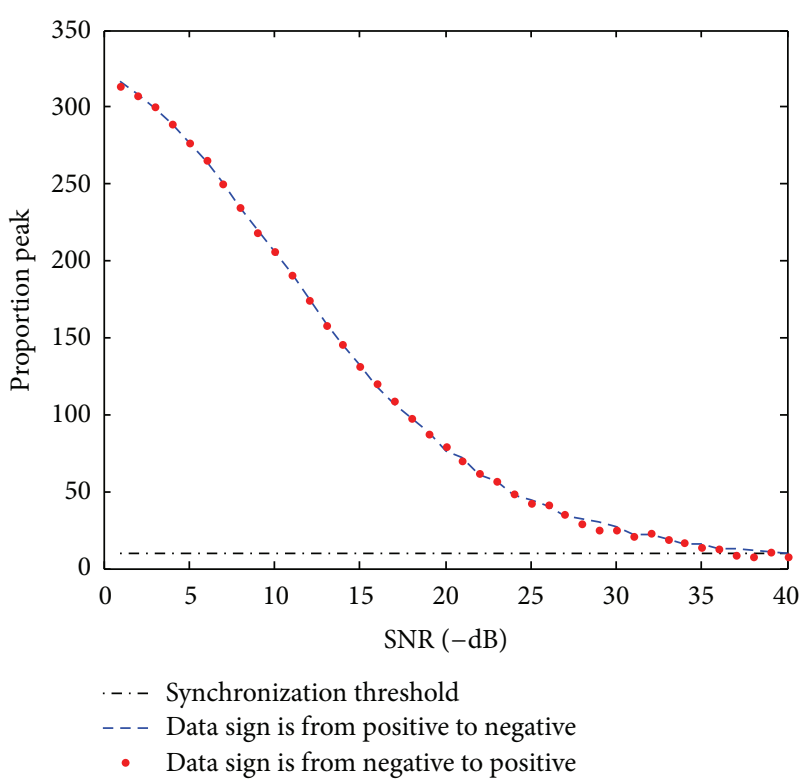

FIGURE 4: The relationship of the synchronization proportion peak and the SNR.

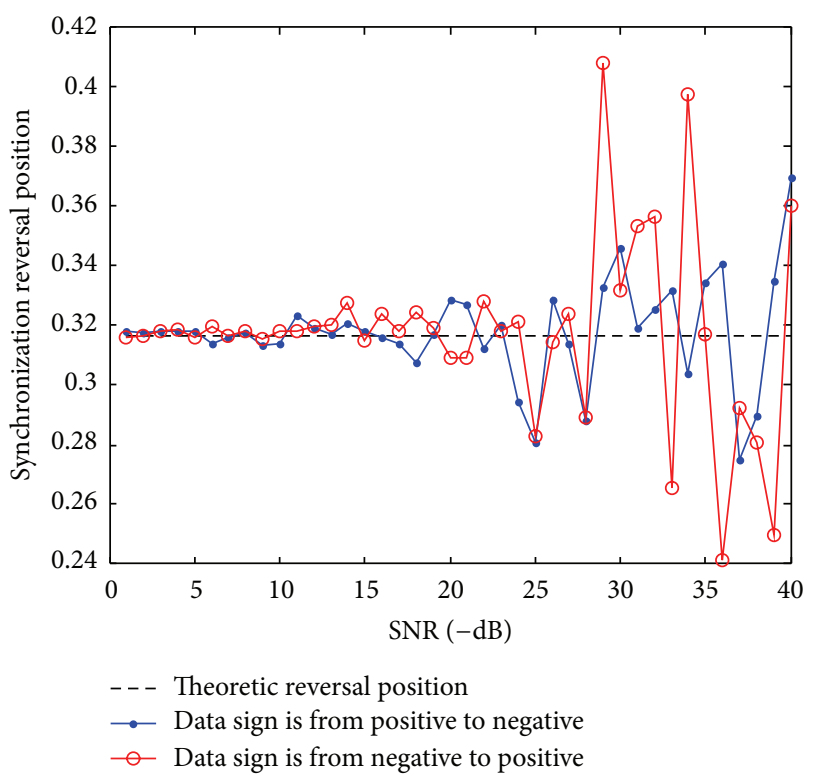

FIGURE 5: The estimated reversal position change with changing SNR.

is tested. With changing SNR, the changes of the estimated reversal position and the theoretical reversal position are shown in Figure 5, revealing that the estimated value increases gradually with decreasing SNR. Next, the absolute value of the estimated reversal error is shown in Figure 6, the result of which indicates that the error increases clearly when SNR is less than $-25 \mathrm{~dB}$.

3.2. The Comparison Analysis of the Algorithms. To verify the superiority of the new algorithm, it is compared with other algorithms, namely, the PN algorithm and the FZ algorithm.

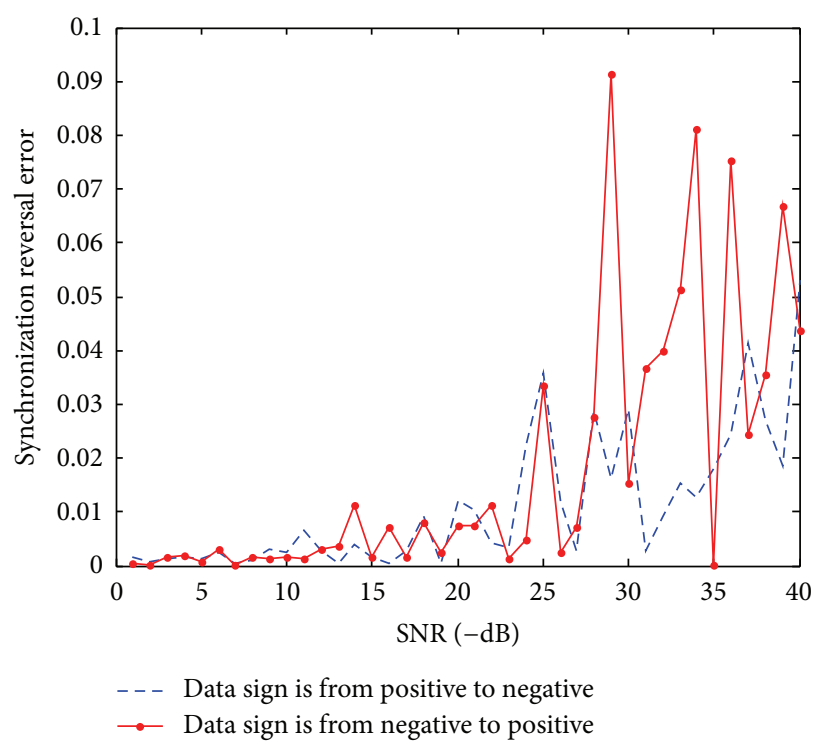

FIGURE 6: The estimated reversal error change with changing SNR.

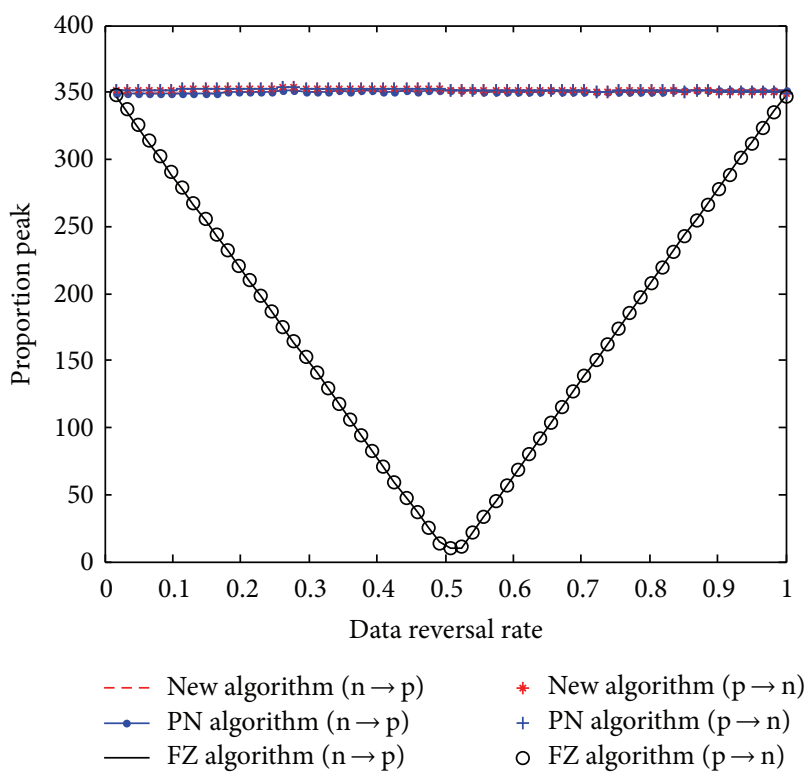

FIGURE 7: The proportion peak comparison with changing data reversal rate.

The parameter $\mathrm{n} \rightarrow \mathrm{p}$ represents that the data sign is from negative to positive, and $\mathrm{p} \rightarrow \mathrm{n}$ represents that the data sign is from positive to negative. With changing data reversal rate, the proportion peak comparison is shown in Figure 7. The result shows that the FZ algorithm's proportion peak is the smallest when the data reversal position is in the middle of the received signal, whereas the proportion peaks of the new algorithm and the PN algorithm are both higher. With changing data reversal rate, the estimated reversal errors comparison is shown in Figure 8. The result shows that the FZ algorithm's estimated reversal error is the highest, and the new algorithm's estimated reversal error is the lowest, 


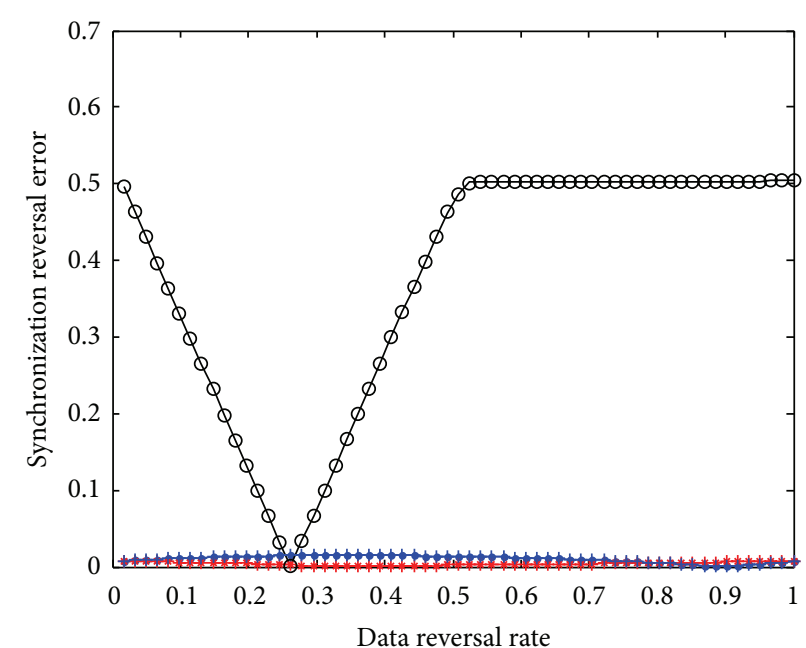

$$
\begin{array}{ll}
-- \text { New algorithm }(n \rightarrow p) & * \text { New algorithm }(p \rightarrow n) \\
\rightarrow-\text { PN algorithm }(n \rightarrow p) & + \text { PN algorithm }(p \rightarrow n) \\
- \text { FZ algorithm }(n \rightarrow p) & \circ \text { FZ algorithm }(p \rightarrow n)
\end{array}
$$

FIgURE 8: The estimated reversal errors comparison with changing data reversal rate.

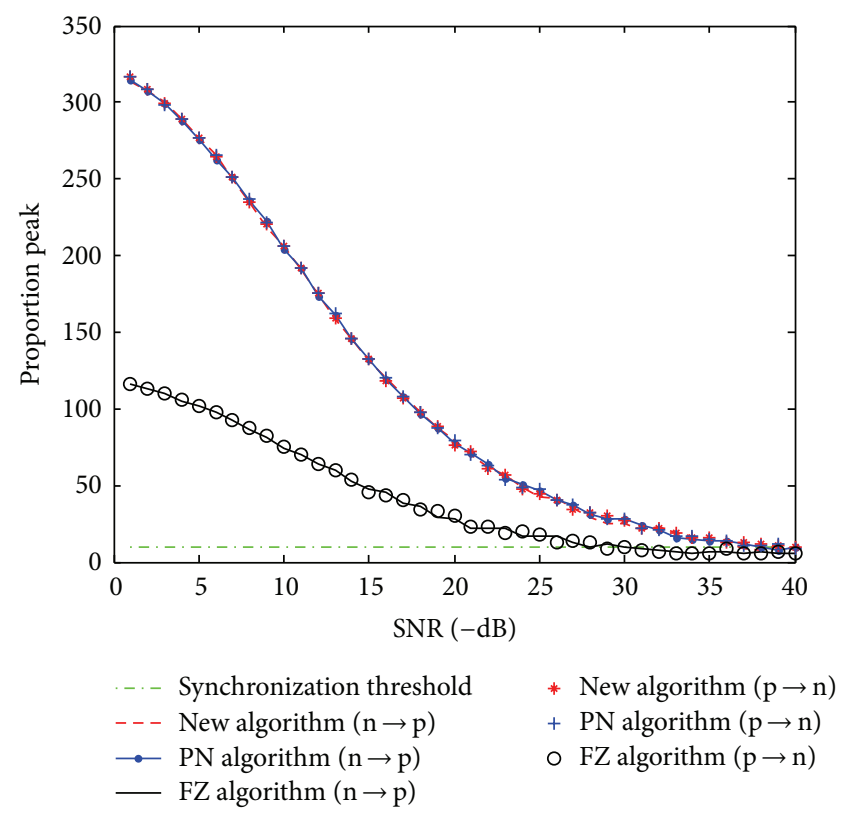

FIGURE 9: The proportion peak comparison with changing SNR.

demonstrating that the new algorithm's synchronization performance is the best.

With changing SNR, the proportion peak comparison is shown in Figure 9. The results show that the FZ algorithm's proportion peak is the smallest under the same SNR condition, but there are no significant differences between the new algorithm and the PN algorithm. And the FZ algorithm can achieve the threshold required when the SNR is less than $-29 \mathrm{~dB}$, but the new algorithm can achieve the threshold required when the SNR is less than $-37 \mathrm{~dB}$. Furthermore, the estimated reversal errors comparison is

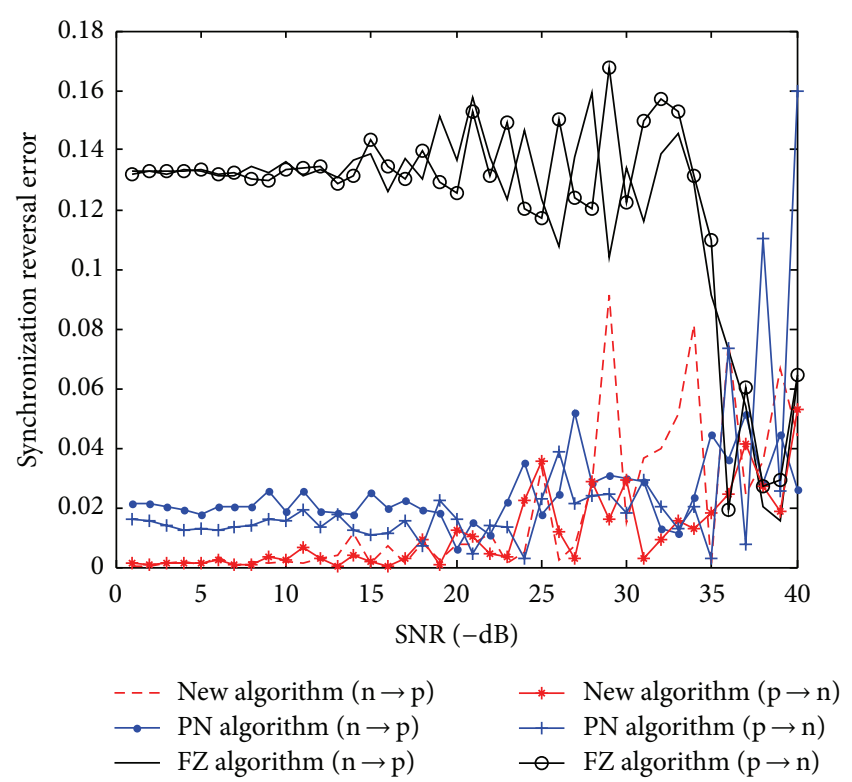

FIgURE 10: The estimated reversal errors compared with changing SNR.

shown in Figure 10, revealing that the estimated reversal errors of three algorithms increase gradually with decreasing SNR. The errors of the FZ algorithm, the PN algorithm, and the new algorithm obviously increase when the SNR is less than $-15 \mathrm{~dB},-23 \mathrm{~dB}$, and $-25 \mathrm{~dB}$, respectively. Thus, the results show that the new algorithm's estimated reversal error is the smallest, and the FZ algorithm's error is the largest, demonstrating that the new algorithm's applicability is the best.

\section{Conclusions}

In this paper, the principle and characteristics of TDDM signals were studied, and the effective algorithms were studied, including the filled zero (FZ) algorithm and the positivenegative (PN) algorithm. Considering the deficiency problems in accuracy and environment adaptability for traditional algorithms, we proposed the optimization synchronization algorithm with illegibility elimination. We eliminated the data inversion illegibility threat by structuring special local sequence and establishing inversion estimation formula. In addition, we improved the correlation judgment mechanism by multicorrelation processing and proportion threshold processing. We demonstrated the new algorithm's correlation precision and SNR applicability by simulation analysis. In addition, we demonstrated that the new algorithm is better than the traditional algorithms in synchronization accuracy and adaptability.

\section{Competing Interests}

The authors declare that they have no competing interests. 


\section{Acknowledgments}

This work was supported by the National Natural Science Foundation of China (no. 61501309) and China Postdoctoral Science Foundation (no. 2015M580231).

\section{References}

[1] J. K. Holmes and S. Raghavan, "A summary of the new GPS IIR$\mathrm{M}$ and IIF modernization signals," in Proceedings of the IEEE 60th Vehicular Technology Conference (VTC '04), pp. 4116-4126, September 2004.

[2] J. J. H. Wang, "Antennas for global navigation satellite system (GNSS)," Proceedings of the IEEE, vol. 100, no. 7, pp. 2349-2355, 2012.

[3] F. Vejrazka, "Galileo and the other satellite navigation systems," in Proceedings of the 17th International Radioelektronika Conference, pp. 1-4, IEEE, Brno, Czech Republic, 2007.

[4] O. Luba, L. Boyd, A. Gower, and J. Crum, "GPS III system operations concepts," IEEE Aerospace and Electronic Systems Magazine, vol. 20, no. 1, pp. 10-18, 2005.

[5] P. G. Mattos and F. Pisoni, "GPS-III L1C signal reception demonstrated on QZSS," in Proceedings of the IEEE/ION Position, Location and Navigation Symposium (PLANS '12), pp. 11621168, IEEE, Myrtle Beach, SC, USA, April 2012.

[6] F. Martín, S. D’Addio, A. Camps, and M. Martín-Neira, “Modeling and analysis of GNSS-R waveforms sample-to-sample correlation," IEEE Journal of Selected Topics in Applied Earth Observations and Remote Sensing, vol. 7, no. 5, pp. 1545-1559, 2014.

[7] S. U. Qaisar and A. G. Dempster, "Assessment of the GPS L2C code structure for efficient signal acquisition," IEEE Transactions on Aerospace and Electronic Systems, vol. 48, no. 3, pp. 1889-1902, 2012.

[8] M. L. Psiaki, B. W. O'Hanlon, J. A. Bhatti, D. P. Shepard, and T. E. Humphreys, "GPS spoofing detection via dual-receiver correlation of military signals," IEEE Transactions on Aerospace and Electronic Systems, vol. 49, no. 4, pp. 2250-2267, 2013.

[9] X. Yu, Y. Sun, J. Liu, and J. Miao, "Design and realization of synchronization circuit for GPS software receiver based on FPGA," Journal of Systems Engineering and Electronics, vol. 21, no. 1, pp. 20-26, 2010.

[10] X. Li and W. Guo, "Efficient differential coherent accumulation algorithm for weak GPS signal bit synchronization," IEEE Communications Letters, vol. 17, no. 5, pp. 936-939, 2013.

[11] T. H. Ta, N. C. Shivaramaiah, A. G. Dempster, and L. L. Presti, "Significance of cell-correlation phenomenon in GNSS matched filter acquisition engines," IEEE Transactions on Aerospace and Electronic Systems, vol. 48, no. 2, pp. 1264-1286, 2012.

[12] C.-W. Chen, S.-H. Chen, H.-W. Tsao, and W.-L. Mao, "Memorybased two-dimensional-parallel differential matched filter correlator for global navigation satellite system code acquisition," IET Radar, Sonar \& Navigation, vol. 8, no. 5, pp. 525-535, 2014.

[13] H. Li, M. Lu, and Z. Feng, "Partial-correlation-result reconstruction technique for weak global navigation satellite system long pseudo-noise-code acquisition," IET Radar, Sonar \& Navigation, vol. 5, no. 7, pp. 731-740, 2011.

[14] C.-J. Li, M.-Q. Lu, Z.-M. Feng, and Q. Zhang, "Study on GPS L2C acquisition algorithm and performance analysis," Journal of Electronics \& Information Technology, vol. 32, no. 2, pp. 296300,2010 .
[15] F. Liu and Y. Feng, "A long code acquisition algorithm on resolve time-frequency uncertainty problem," Acta Aeronautica et Astronautica Sinica, vol. 34, no. 8, pp. 1924-1933, 2013. 


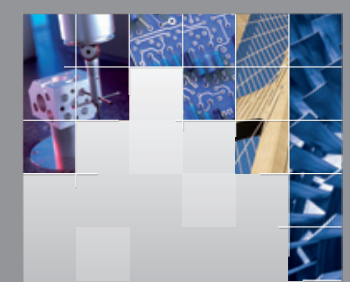

\section{Enfincering}
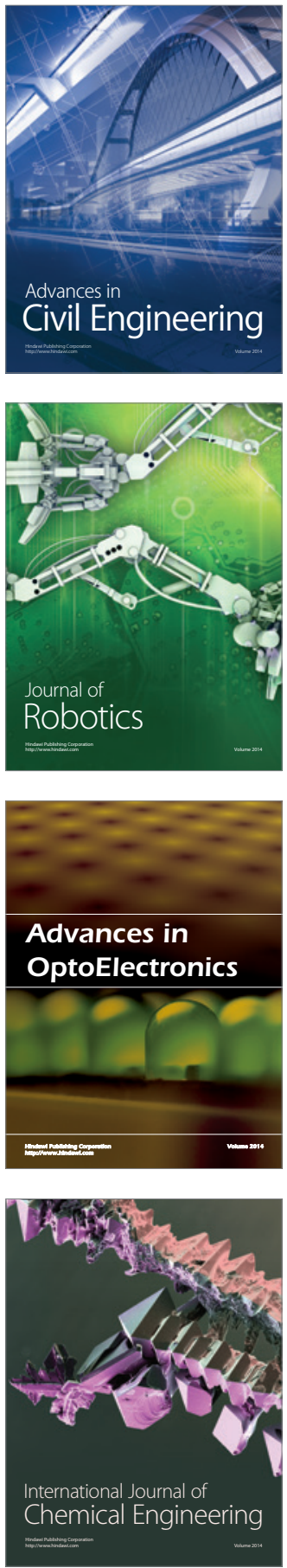

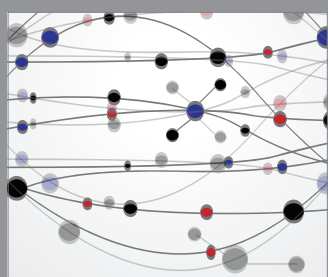

The Scientific World Journal

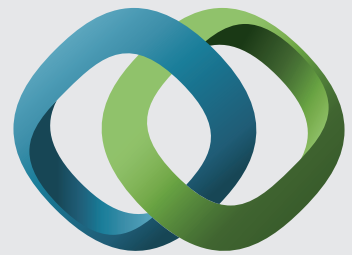

\section{Hindawi}

Submit your manuscripts at

http://www.hindawi.com
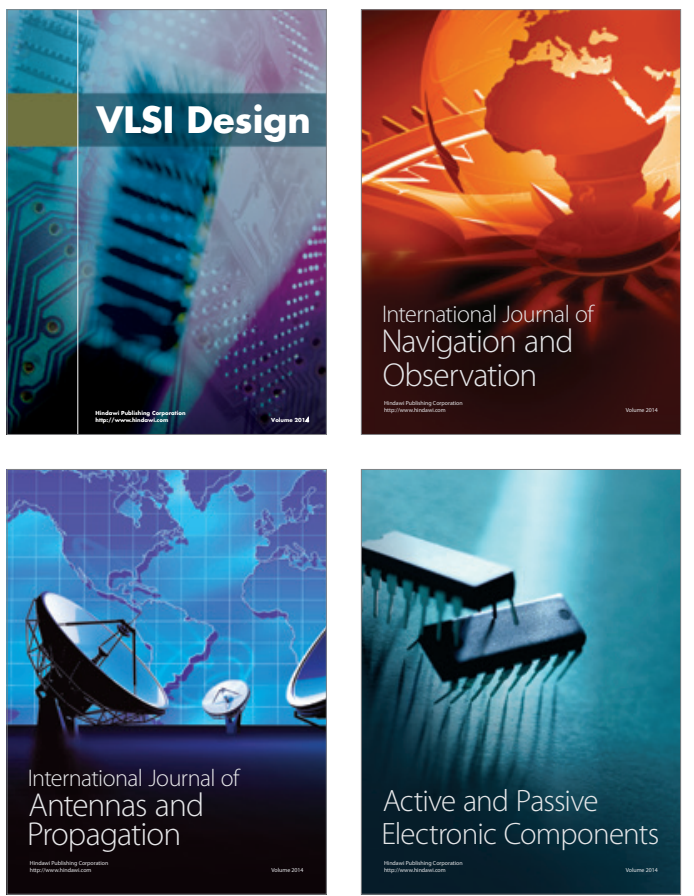
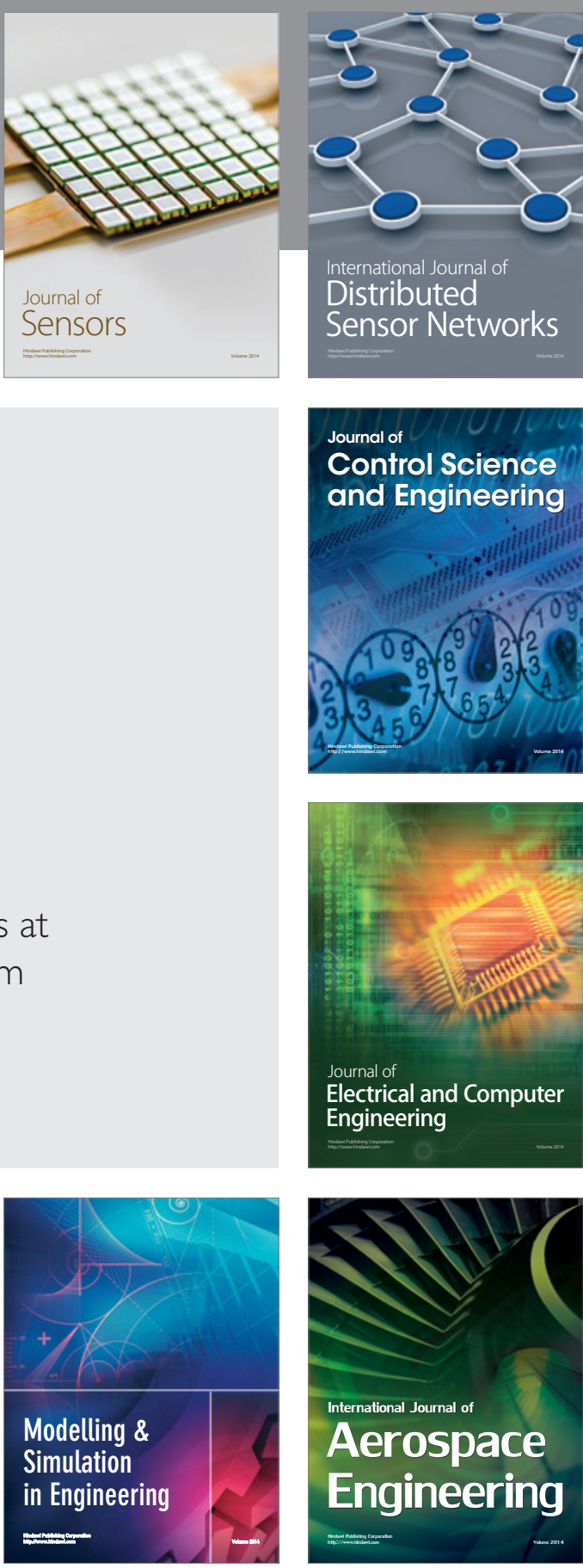

International Journal of

Distributed

Sensor Networks

Journal of

Control Science

and Engineering
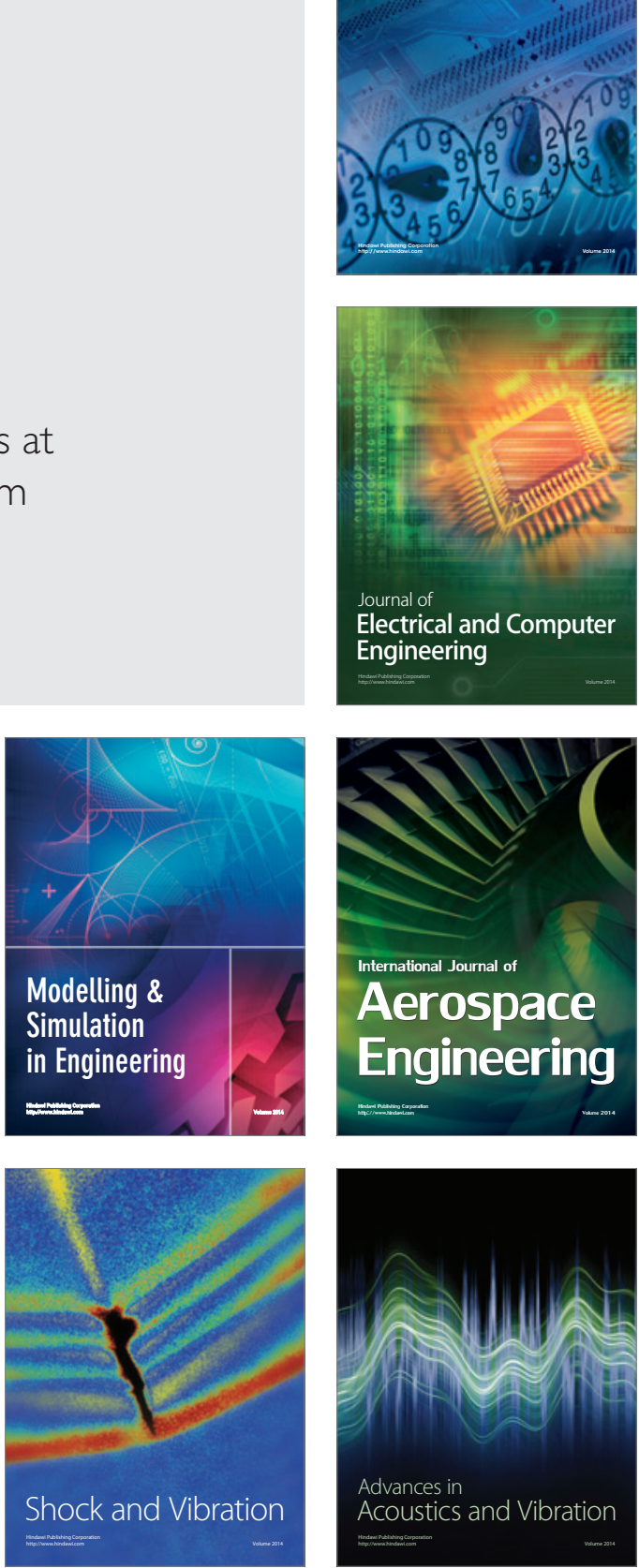